\title{
Pembrolizumab-induced myocarditis in a patient with malignant mesothelioma: plasma exchange as a successful emerging therapy-case report
}

\author{
Sanziana R. I. Schiopu ${ }^{1,2}$, Lukas Käsmann ${ }^{3,4}$, Ulf Schönermarck ${ }^{5}$, Michael Fischereder ${ }^{5}$, \\ Ulrich Grabmaier ${ }^{1,2}$, Farkhad Manapov ${ }^{3,4}$, Josefine Rauch ${ }^{3,4}$, Martin Orban ${ }^{1,2}$ \\ ${ }^{1}$ Intensive Care Unit, Medical Clinic I, Ludwig Maximilian University Munich, Munich, Germany; ${ }^{2}$ Department of Cardiology, DZHK (German \\ Center for Cardiovascular Research), Partner Site Munich Heart Alliance, Munich, Germany; ${ }^{3}$ Department of Radiation Oncology, University \\ Hospital, Ludwig Maximilian University Munich, Munich, Germany; ${ }^{4}$ Comprehensive Pneumology Center Munich (CPC-M), Munich, Germany; \\ ${ }^{5}$ Medical Clinic IV, Ludwig Maximilian University Munich, Munich, Germany \\ Correspondence to: Sanziana R. I. Schiopu, MD. Medizinische Klinik I, LMU Klinikum, Marchioninistrasse 15, 81377 Munich, Germany. \\ Email: sanziana.schiopu@med.uni-muenchen.de.
}

\begin{abstract}
Malignant mesothelioma is an aggressive cancer associated with prior exposure to asbestos and dismal prognosis. Immune checkpoint inhibitor therapy is currently approved by the Food and Drug Administration for pre-treated malignant pleural mesothelioma. We describe a 75 -year-old patient with disseminated, progressive malignant mesothelioma receiving 2 cycles of pembrolizumab who presented with generalized muscle weakness, shortness of breath, double vision and ptosis. There was no previous history of cardiovascular disease. The clinical picture, supported by the detection of anti-titin autoantibodies suggested myasthenia gravis (MG). Also, cardiac biomarkers were elevated. Echocardiography showed new severely reduced ejection fraction. A 12-lead resting electrocardiogram (ECG) revealed ST segment elevation in the posterior leads with polymorphic ventricular extrasystoles. Because cardiac catheterization revealed no relevant coronary lesions, immune checkpoint inhibitor-associated myocarditis and MG were suspected. Management and Outcome: The patient was started on steroids. Within a few days of presentation respiratory failure set in and the patient was intubated. Recurrent arrhythmias followed, which were treated by repeated emergency electrical cardioversion. In order to relieve myasthenic symptoms, plasma exchange was initiated and 10 cycles were carried out. This consequently also led to an improvement of myocarditis. Upon discharge, the ejection fraction recovered. The patient recovered and was alive at 1-year follow-up, without relevant limitations to his quality of life. Discussion and Conclusion: The article further discusses the use of plasma exchange for immune checkpoint inhibitor-associated myocarditis based on a review of literature. We conclude that patients showing no improvement after steroid therapy for immune checkpoint inhibitor-related myocarditis should be evaluated for plasma exchange, which appears to be an effective treatment option.
\end{abstract}

Keywords: Mesothelioma; myocarditis; immune check inhibition; plasma exchange; treatment related toxicity

Submitted Oct 12, 2020. Accepted for publication Dec 23, 2020.

doi: $10.21037 /$ tlcr-20-1095

View this article at: http://dx.doi.org/10.21037/tlcr-20-1095

\section{Introduction}

Malignant mesothelioma is an aggressive cancer associated with prior exposure to asbestos and dismal prognosis. While asbestos has been banned in the western world, the incidence of malignant pleural mesothelioma does not appear to drop (1). Treatment options, as put forward by the current guidelines, consist of surgery, chemotherapy and/or radiotherapy, but clear recommendations exist only for first 
line therapy (2). There is however no consensus on secondline therapy in previously treated and relapsed malignant pleural mesothelioma $(2,3)$. Immunotherapy can be offered to patients upon enrollment in clinical trials, either as combined or monotherapy $(4,5)$. Due to promising results in trials and good tolerance, their use continues to extend to other types of cancer as well.

Pembrolizumab belongs to the class of immune-checkpoint inhibitors (ICI) and is a humanized IgG4 antibody that blocks the interaction between programmed cell death protein 1 (PD1) and programmed death-ligand 1 (PD-L1). It is indicated in the treatment of various cancer subtypes and is currently approved by the Food and Drug Administration for pre-treated malignant pleural mesothelioma (4).

Among immune-related adverse events (irAEs) of pembrolizumab, myocarditis and neuromuscular adverse events are considered rare. However, evidence for a higher incidence of ICI-associated myocarditis is emerging (6-8). To the best of our knowledge, there is no consensus on diagnostic and treatment management. In this case report we present a patient with recurrent malignant pleural mesothelioma, who developed ICI-related myocarditis, our treatment approach and outcome. We present the following article in accordance with the CARE reporting checklist (available at http://dx.doi.org/10.21037/tlcr-20-1095).

\section{Case presentation}

A 75-year-old gentleman [75 kg, $178 \mathrm{~cm}$, body-mass index $24 \mathrm{~kg} / \mathrm{m}^{2}$, Karnofsky Performance Status (KPS) 90\%] undergoing second-line treatment for malignant mesothelioma was referred from his radiotherapist to our cardiology department with suspicion of acute coronary syndrome. There was no previous history of cardiovascular disease or any relevant comorbidity.

Initially, the patient was admitted to our hospital due to unknown weight loss (12 kg in $<6$ months) and abdominal pain. A positron emission tomography-computed tomography (PET-CT) scan revealed a supradiaphragmal, pleural tumor with diaphragmatic infiltration and suspicious peritoneal lymph node involvement (cT4 cN1 MX). Following current guidelines and a consensual, multidisciplinary decision of the tumor board, cytoreductive surgery with heated intraperitoneal chemotherapy (HIPEC) was initiated. The patient received partial diaphragmatic resection with alloplastic reconstruction. Besides, a subtotal parietal peritonectomy was performed with partial resection of the ribs 9-12, followed by HIPEC. Histopathologic examination revealed a malignant epithelioid mesothelioma: pT2 pN1 (2/5 LK) M0 (UICC stage II). Afterwards a wait-and-see strategy was preferred to chemotherapy (pemetrexed/cisplatin). Staging at 12 months revealed pleural and perihepatic relapse. Nextgeneration sequencing (using Oncomine Precision Assay, Thermo Fisher Scientific, USA) revealed no mutations within the tumor cell genome. Stereotactic body radiotherapy was applied to the basal and lateral pleural metastases. Interstitial brachytherapy was applied to liver metastasis. A positron emission tomography - computed tomography routine control at 24 months revealed progressive disease with multiple new bipulmonary and lymph node metastases with diffuse, extensive spread throughout the abdomen.

Given the progressive disease and considering a PD-L1 expression of $5 \%$, anti-PD1 agent pembrolizumab with a dosage of $200 \mathrm{mg}$ every 3 weeks, was initiated in a compassionate use program with good tolerance of the first cycle. A second cycle of pembrolizumab was scheduled. Shortly after the second cycle, the patient reported an alteration of his general state accompanied by generalized muscle weakness, shortness of breath, double vision and ptosis. The patient was immediately admitted to our hospital.

\section{Complications and admission}

A transthoracic echocardiography showed severely reduced ejection fraction at time of admission. A 12-lead resting electrocardiogram (ECG) revealed ST segment elevation in the posterior leads with polymorphic ventricular extrasystoles (Figure 1). Laboratory parameters are displayed in Table 1. Creatinine $1,1 \mathrm{mg} / \mathrm{dL}$ (normal range, $0.7-1.2 \mathrm{mg} / \mathrm{dL}$ ) and hemoglobin 14,8 g/dL (normal range, $13.5-17.5 \mathrm{~g} / \mathrm{dL}$ ) were within normal range. Cardiac catheterization revealed no relevant coronary lesions (Figure 2).

With respect to elevated transaminases, serological tests of hepatitis virus (A-E) were negative and imaging showed no evidence for new metastases of the liver, no parenchymal or vessel anomalies or cholecystitis.

The clinical picture with muscle weakness, double vision and ptosis guided towards the differential diagnosis of MG, which was supported by the detection of antititin autoantibodies (Ratio 3.24, normal range $<1.00$ ). Autoantibodies for anti-acetylcholine receptor or musclespecific tyrosine kinase were negative, as well as antibodies for neuronal nuclei or any antibodies specific for paraneoplastic or autoimmune disease [Purkinje Cell, Yo, Amphiphysin, 


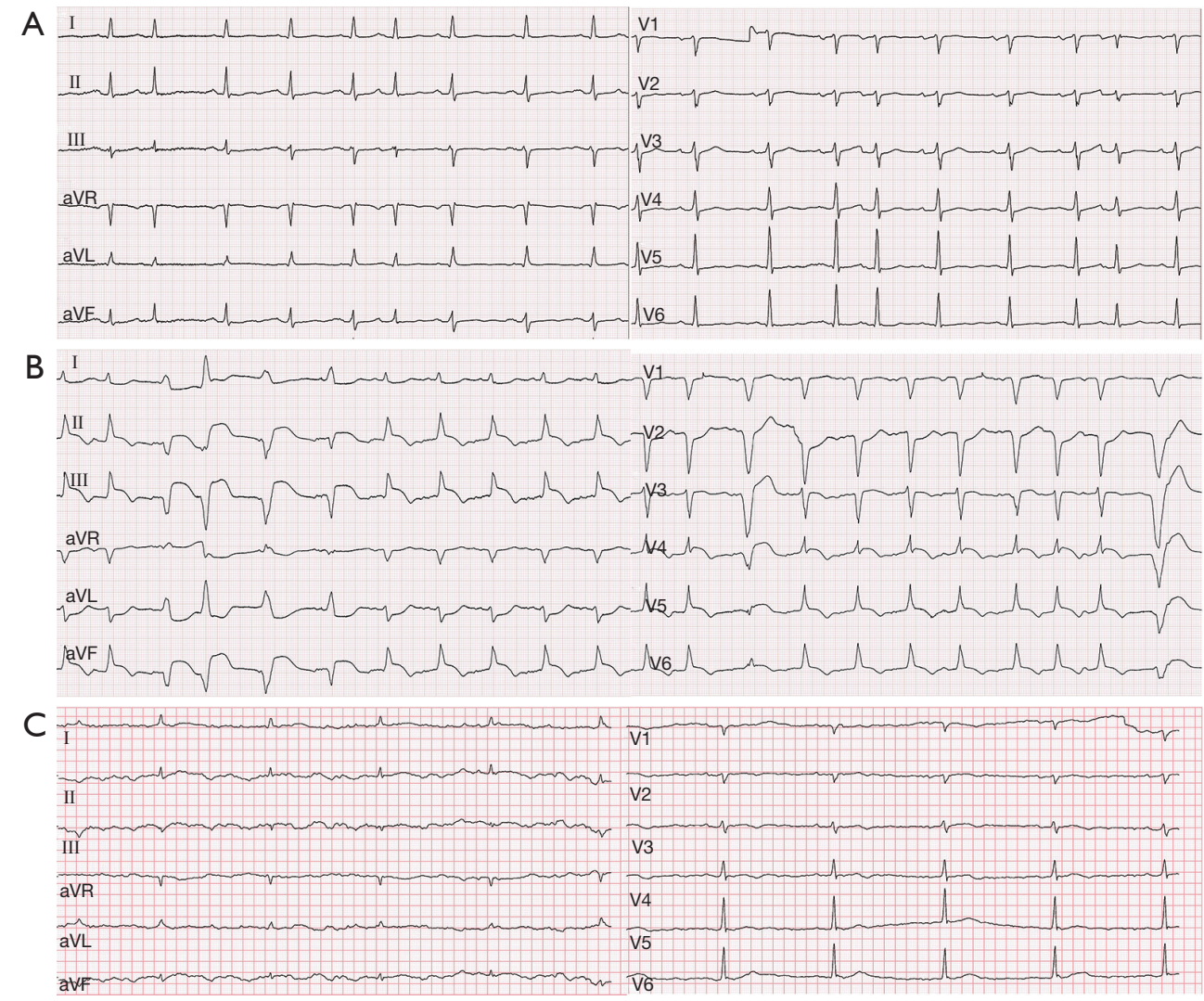

Figure 1 12-lead resting electrocardiogram (ECG) prior to immunotherapy (A), showing unremarkable findings with supraventricular extrasystoles. ECG upon hospital admission (B) revealing ST segment elevation in the posterior leads with polymorphic ventricular extrasystoles. ECG upon discharge from our intensive care unit $(\mathrm{C})$.
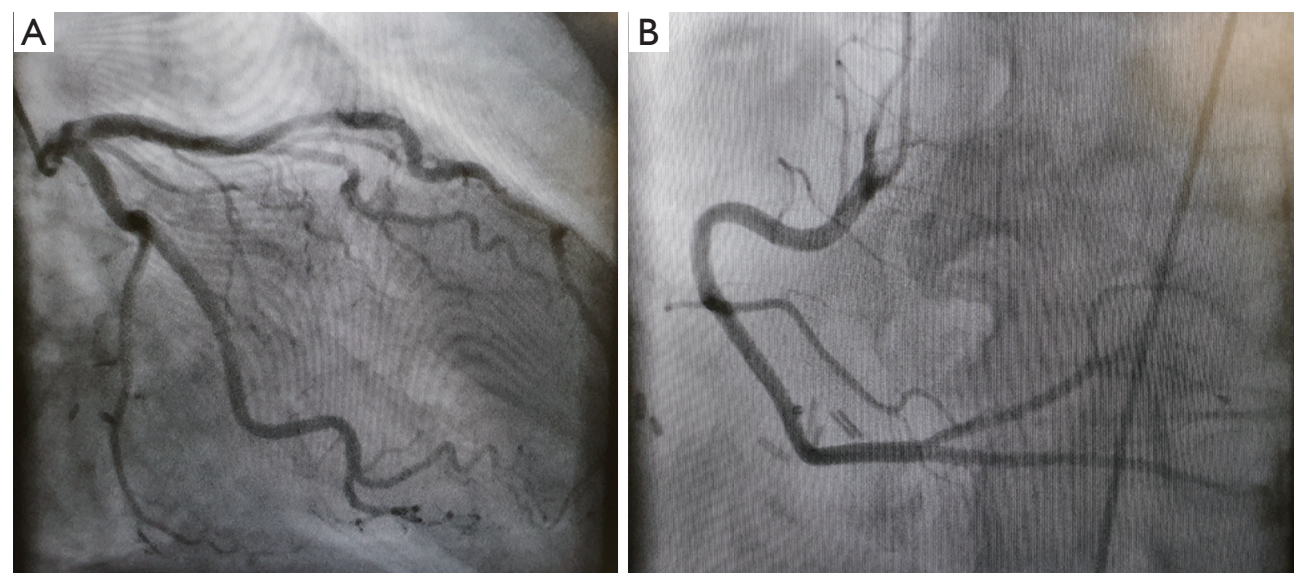

Figure 2 Coronary angiogram showing normal coronary arteries without evidence of relevant coronary artery disease. (A) left coronary artery. (B) Right coronary artery. 
Table 1 Laboratory parameters from starting point of immunotherapy (pembrolizumab) to onset of symptoms, hospital admission and ICU treatment

\begin{tabular}{|c|c|c|c|c|c|c|c|}
\hline Parameter & AST (U/L) & ALT (U/L) & Gamma-GT (U/L) & $\mathrm{LDH}(\mathrm{U} / \mathrm{L})$ & CK (U/L) & CK-MB activity (U/L) & Troponin $\mathrm{T}(\mathrm{ng} / \mathrm{mL})$ \\
\hline Pembrolizumab $1^{\text {st }}$ cycle & 26 & 31 & 77 & 220 & & & \\
\hline Pembrolizumab $2^{\text {nd }}$ cycle & 134 & 66 & 82 & 486 & & & \\
\hline \multicolumn{8}{|l|}{ Hospital admission } \\
\hline Day 2 & 288 & 461 & 198 & 1,180 & 3,480 & 204 & 3.02 \\
\hline Day 3 & 239 & 496 & 343 & 960 & 2,488 & 127 & 4.82 \\
\hline Day 6 & 112 & 237 & 246 & & 1,477 & 45 & \\
\hline Day 9 & 63 & 143 & 106 & & 577 & 44 & \\
\hline
\end{tabular}

ICU, intensive care unit; AST, aspartate transaminase; ALT, alanine transaminase; GT, glutamyl transferase; LDH, lactate dehydrogenase; $\mathrm{CK}$, creatine kinase; CK-MB, creatine kinase-myocardial band.

NMDA, AMPA1, AMPA2, CASPR2, LGI1, GABA B(1/2)Receptor, Hu, Ri, Ma2(Ta), Ma1, CV2(CRMP5)].

The clinical presentation, laboratory results and medical history were suggestive of ICI-associated myocarditis. In addition, the patient suffered from ICI-associated MG and hepatitis.

The patient was immediately started on steroids (intravenous methylprednisolone, $100 \mathrm{mg} / \mathrm{day}$, approx. $1.5 \mathrm{mg} / \mathrm{kg}$ ) at the time of admission. However, due to acute respiratory failure following $M G$, he was transferred to our intensive care unit 72 hours later and non-invasive ventilation (NIV) was initiated. Since vigilance impairment quickly progressed to coma (Glasgow Coma Scale 3), the patient was intubated. A CT scan of the brain, thorax and abdomen was performed. There was no evidence for edema, new metastases, stroke or intracranial bleeding; neither for pneumonitis nor pulmonary embolism. Furthermore, infectious or neoplastic encephalitis could be excluded (brain MRI, cerebrospinal fluid sampling). Considering the progression of respiratory and hemodynamic compromise, we did not pursue endomyocardial biopsy, due to its invasiveness. Also, we were not able to perform cardiac MRI.

The patient responded well to catecholamines and could be stabilized. Catecholamines and ventilation pressures could be reduced. Forty-eight hours later the patient could be extubated and tapering of steroids was conducted. Because of persistent hypercapnia NIV was continued. Twenty-four hours later the patient developed recurrent arrhythmias (atrial fibrillation and atrial flutter) with hemodynamic compromise, which were treated by repeated emergency electrical cardioversion. Therapeutic anticoagulation was initiated. While transaminases plateaued, neurological symptoms and cardiotoxicity (Common Terminology Criteria of Adverse Events Grade 4) proved refractory to our therapy. Thus, in interdisciplinary discussion with nephrologists and haemato-oncologists, plasma exchange was initiated to relieve neurological symptoms and ten cycles were carried out. Vigilance and level of consciousness, as well as respiratory and cardiac function improved. Daily requirement of NIV intervals could be steadily reduced. Echocardiography documented an improvement of the ejection fraction (moderately reduced, $45-50 \%)$. An overview of the patient's clinical course and management is displayed in Figure 3.

Unfortunately, recurrent ventricular arrhythmias led to hemodynamic compromise on day 40 (since the first administration of pembrolizumab) and the patient was reintubated and reventilated again. Sinus rhythm could be restored by electrical and pharmacological cardioversion. The patient improved once again and weaning could be initiated. A timeline of events is depicted in Table S1. 


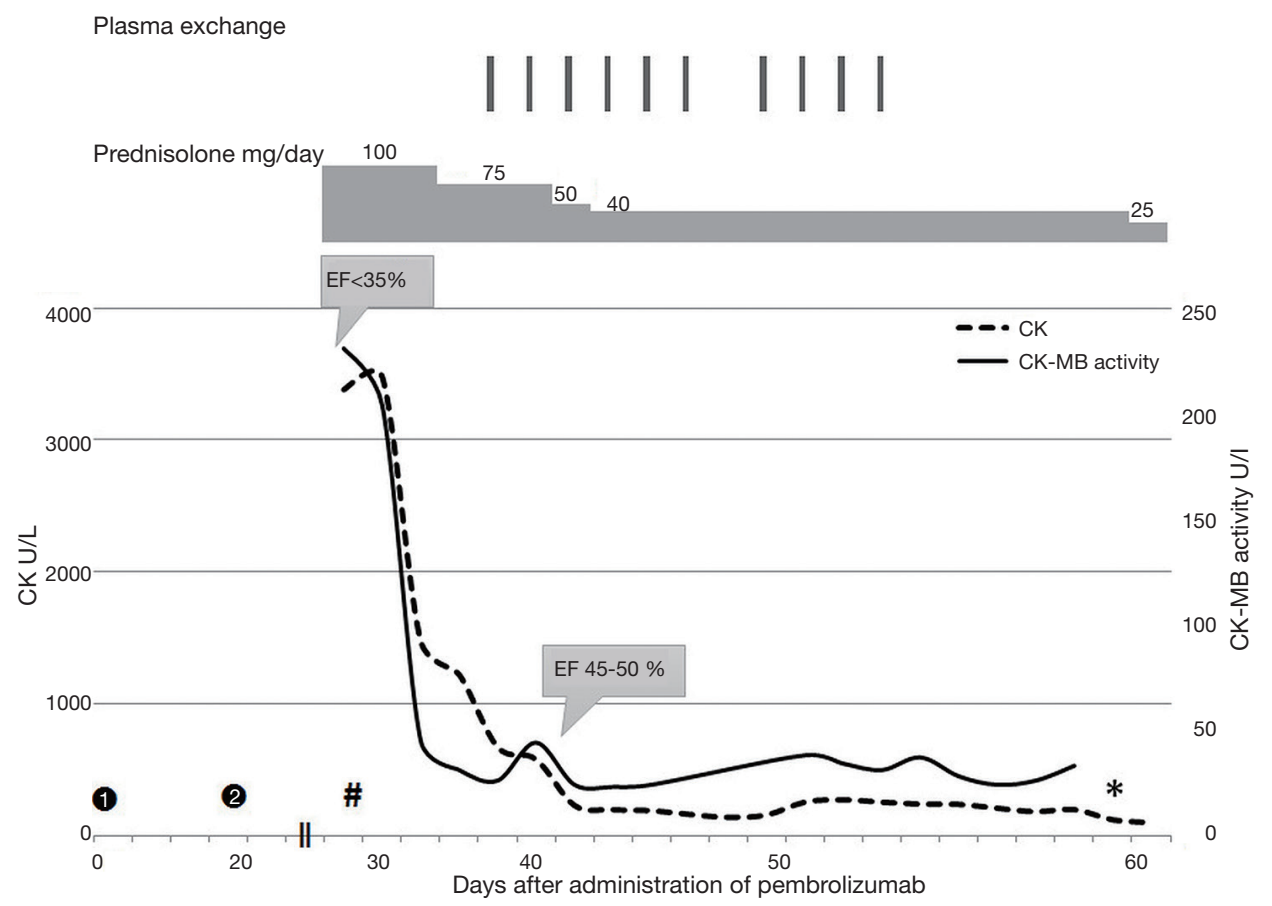

Figure 3 Clinical course of a 75 -year-old man with myocarditis and myasthenia gravis after administration of pembrolizumab. Creatinine kinase and creatinine kinase-myocardial band activity were increased after the second dose of pembrolizumab. 1 , first dose of pembrolizumab; 2, second dose of pembrolizumab; \#, hospital admission; *, discharge.

\section{Discharge and follow-up}

Approximately 1 month after the onset of symptoms, the patient could be transferred to an external weaning ward. He recovered and was appointed for outpatient care. Due to grade 4 toxicity, pembrolizumab was discontinued. Progression-free survival amounted to about 1 year, during which the patient enjoyed a good quality of life without relevant limitations (KPS 70\%). The ejection fraction recovered (mildly reduced, approx. 50-55\%), as assessed by transthoracic echocardiography. A 12-lead resting ECG showed sinus rhythm with extrasystoles. A CT scan 12 months after the last treatment showed progressive disease. The patient ultimately passed away due to respiratory failure.

\section{Ethical statement}

All procedures performed in studies involving human participants were in accordance with the ethical standards of the institutional and/or national research committee(s) and with the Helsinki Declaration (as revised in 2013). Written informed consent was obtained from the patient and is available upon request.

\section{Discussion}

We report on a patient with disseminated, progressive malignant mesothelioma, treated with pembrolizumab, who developed ICI-associated MG, myocarditis with hemodynamic compromise and hepatitis. The decision of administering pembrolizumab was based on the promising trial results of NCT02054806 (KEYNOTE-028) (9) and NCT02399371 (10). In the malignant mesothelioma cohort of KEYNOTE-028, an objective response rate (ORR) of $20 \%$ and median response duration of 12 months were observed, similar to the ORR of $22 \%$ and median overall survival of 11.5 months in NCT02399371. However, the results of a randomized phase III trial (NCT02991482, PROMISE-meso) (11) found no difference in median PFS or OS (median OS of 10.7 months for pembrolizumab versus 11.7 months for gemcitabine or vinorelbine). Although pembrolizumab resulted in higher ORR compared to chemotherapy (22\% versus $6 \%$ ). In summary, selected patients may benefit from pembrolizumab treatment, but further studies are warranted to identify these patients. 
To the best of our knowledge, this is the first case of ICIrelated myocarditis in a mesothelioma patient, with no previous history of cardiovascular disease, where plasma exchange was initiated and proved effective, after failure of immunosuppressive agents (steroids).

\section{Severe side effects of ICI}

ICI immune-related adverse effects are organ-specific, with dermatologic, gastrointestinal, endocrine, respiratory and hepatic effects being the most commonly reported in clinical trials (12). ICI-associated myocarditis, while rare, is fulminant and often fatal (13).

\section{Standard treatment}

Steroids constitute the usual therapeutic approach to immune-related adverse effects, but recommendations vary widely with respect to dose and timing $(14,15)$. In a recent retrospective study, higher initial steroid dose (i.e., intravenous methylprednisolone $1,000 \mathrm{mg} / \mathrm{d}$ ) and earlier initiation (within 24 hours of admission) were associated with improved cardiac outcomes with ICI-associated myocarditis (16). However, corticosteroids may reduce the efficacy of PD-L1 blockade and therefore may have a negative effect on the efficacy of ICI. Data to support this is available from patients with non-small cell lung cancer and melanoma, who are most often treated with ICI $(17,18)$. Steroids have been associated with poorer outcome and decreased progression-free survival in these patients and therefore there is a need for therapies beyond corticosteroids.

\section{An emerging therapy}

In a review of ICI-associated myocarditis, only 3 out of 42 case reports from 2015-2018 described the use of plasma exchange (19). Table S2 presents a selection of case reports on ICI-associated myocarditis, where plasma exchange was used as part of multimodal therapy, leading to clinical improvement in most cases. Plasma exchange is best known in neurological practice and to the best of our knowledge there is so far no guideline on plasma exchange in the management of ICI-related myocarditis (20,21). A drawback of plasma exchange is that it is usually only available in expert medical centers. In the case of our patient, there had been no significant neurological or cardiovascular improvement using steroids alone. Considering the new MG symptoms and based on evidence from neurological practice (22), we initiated relatively early plasma exchange. This consequently led to an improvement not only of MG, but also of myocarditis. For this reason, we recommend plasma exchange as early as possible, although the exact mechanisms still remain unclear. Possible effects of plasma exchange may be either the removal auf auto-antibodies, immune complexes and cytokines and/or the fast reduction of the drug levels (i.e., pembrolizumab), as has been shown for other monoclonal antibodies (23).

\section{Conclusions}

ICI-associated myocarditis is considered a rare adverse effect and current knowledge is retrieved from single case reports and small case series. Cardiac monitoring (e.g., with ECG or assessment of troponin levels) is not routinely performed in most immunotherapy trials, therefore the true incidence remains unknown. We suggest, patients showing no improvement after steroid therapy for ICI-related myocarditis to be timely evaluated for plasma exchange, which appears to be an effective treatment option. Further research should be undertaken to understand the underlying mechanisms, identify patients at risk and establish treatment protocols for ICI-related myocarditis.

\section{Acknowledgments}

Funding: None.

\section{Footnote}

Reporting Checklist: The authors have completed the CARE reporting checklist. Available at http://dx.doi.org/10.21037/ tlcr-20-1095

Conflicts of Interest: All authors have completed the ICMJE uniform disclosure form (Available at http://dx.doi. org/10.21037/tlcr-20-1095). MO received speaker honoraria and travel compensations from Abbott Medical, AstraZeneca, Abiomed, Bayer vital, BIOTRONIK, Bristol-Myers Squibb, CytoSorbents, Daiichi Sankyo Deutschland, Edwards Lifesciences Services, Sedana Medical, outside the submitted work. U Schönermarck reports grants, personal fees and nonfinancial support from Alexion, as well as grants and personal fees from Chemocentryx, outside the submitted work. The other authors have no conflicts of interest to declare. 
Ethical Statement: The authors are accountable for all aspects of the work in ensuring that questions related to the accuracy or integrity of any part of the work are appropriately investigated and resolved. All procedures performed in studies involving human participants were in accordance with the ethical standards of the institutional and/or national research committee(s) and with the Helsinki Declaration (as revised in 2013). Written informed consent was obtained from the patient and is available upon request.

Open Access Statement: This is an Open Access article distributed in accordance with the Creative Commons AttributionNonCommercial-NoDerivs 4.0 International License (CC BYNC-ND 4.0), which permits the non-commercial replication and distribution of the article with the strict proviso that no changes or edits are made and the original work is properly cited (including links to both the formal publication through the relevant DOI and the license). See: https://creativecommons. org/licenses/by-nc-nd/4.0/.

\section{References}

1. Carbone M, Adusumilli PS, Alexander HR, Jr., et al. Mesothelioma: Scientific clues for prevention, diagnosis, and therapy. CA Cancer J Clin 2019;69:402-29.

2. Nicolini F, Bocchini M, Bronte G, et al. Malignant Pleural Mesothelioma: State-of-the-Art on Current Therapies and Promises for the Future. Front Oncol 2020;9:1519.

3. Gray SG, Mutti L. Immunotherapy for mesothelioma: a critical review of current clinical trials and future perspectives. Transl Lung Cancer Res 2020;9:S100-19.

4. Metaxas Y, Rivalland G, Mauti LA, et al. Pembrolizumab as Palliative Immunotherapy in Malignant Pleural Mesothelioma. J Thorac Oncol 2018;13:1784-91.

5. Disselhorst MJ, Baas P. Chemotherapy options versus "novel" therapies: how should we treat patients with malignant pleural mesothelioma. Transl Lung Cancer Res 2020;9:S77-85.

6. Giaccone G, Kim C, Thompson J, et al. Pembrolizumab in patients with thymic carcinoma: a single-arm, singlecentre, phase 2 study. Lancet Oncol 2018;19:347-55.

7. Cho J, Kim HS, Ku BM, et al. Pembrolizumab for Patients With Refractory or Relapsed Thymic Epithelial Tumor: An Open-Label Phase II Trial. J Clin Oncol 2019; 37:2162-70.

8. Mahmood SS, Fradley MG, Cohen JV, et al. Myocarditis in Patients Treated With Immune Checkpoint Inhibitors. J Am Coll Cardiol 2018;71:1755-64.
9. Alley EW, Lopez J, Santoro A, et al. Clinical safety and activity of pembrolizumab in patients with malignant pleural mesothelioma (KEYNOTE-028): preliminary results from a non-randomised, open-label, phase $1 \mathrm{~b}$ trial. Lancet Oncol 2017;18:623-30.

10. Desai A, Karrison T, Rose B, et al. OA08.03 Phase II Trial of Pembrolizumab (NCT02399371) In Previously-Treated Malignant Mesothelioma (MM): Final Analysis. J Thorac Oncol 2018;13:S339.

11. Popat S, Curioni-Fontecedro A, Dafni U, et al. A multicentre randomised phase III trial comparing pembrolizumab versus single-agent chemotherapy for advanced pre-treated malignant pleural mesothelioma: the European Thoracic Oncology Platform (ETOP 9-15) PROMISE-meso trial. Ann Oncol 2020;31:1734-45.

12. Darnell EP, Mooradian MJ, Baruch EN, et al. ImmuneRelated Adverse Events (irAEs): Diagnosis, Management, and Clinical Pearls. Curr Oncol Rep 2020;22:39.

13. Martins F, Sofiya L, Sykiotis GP, et al. Adverse effects of immune-checkpoint inhibitors: epidemiology, management and surveillance. Nat Rev Clin Oncol 2019;16:563-80.

14. Brahmer JR, Lacchetti C, Schneider BJ, et al. Management of Immune-Related Adverse Events in Patients Treated With Immune Checkpoint Inhibitor Therapy: American Society of Clinical Oncology Clinical Practice Guideline. J Clin Oncol 2018;36:1714-68.

15. Caforio AL, Pankuweit S, Arbustini E, et al. Current state of knowledge on aetiology, diagnosis, management, and therapy of myocarditis: a position statement of the European Society of Cardiology Working Group on Myocardial and Pericardial Diseases. Eur Heart J 2013;34:2636-48, 2648a-d.

16. Zhang L, Zlotoff DA, Awadalla M, et al. Major Adverse Cardiovascular Events and the Timing and Dose of Corticosteroids in Immune Checkpoint InhibitorAssociated Myocarditis. Circulation 2020;141:2031-4.

17. Arbour KC, Mezquita L, Long N, et al. Impact of Baseline Steroids on Efficacy of Programmed Cell Death-1 and Programmed Death-Ligand 1 Blockade in Patients With Non-Small-Cell Lung Cancer. J Clin Oncol 2018; 36:2872-8.

18. Faje AT, Lawrence D, Flaherty K, et al. High-dose glucocorticoids for the treatment of ipilimumab-induced hypophysitis is associated with reduced survival in patients with melanoma. Cancer 2018;124:3706-14.

19. Atallah-Yunes SA, Kadado AJ, Kaufman GP, et al. Immune checkpoint inhibitor therapy and myocarditis: a systematic review of reported cases. J Cancer Res Clin Oncol 
2019; 145:1527-57.

20. Padmanabhan A, Connelly-Smith L, Aqui N, et al. Guidelines on the Use of Therapeutic Apheresis in Clinical Practice - Evidence-Based Approach from the Writing Committee of the American Society for Apheresis: The Eighth Special Issue. J. Clin. Apher 2019;34:171-354.

21. Zanatta E, Cozzi M, Marson P, et al. The role of plasma exchange in the management of autoimmune disorders. $\mathrm{Br}$
J Haematol 2019;186:207-19.

22. Sanders DB, Wolfe GI, Benatar M, et al. International consensus guidance for management of myasthenia gravis: Executive summary. Neurology 2016;87:419-25.

23. Gauckler P, Leierer J, Kocher F, et al. Lessons learned from immunoadsorption for hyperviscosity in $\operatorname{IgM}$ multiple myeloma-A case report. J Clin Apher 2020;3 5:227-30.
Cite this article as: Schiopu SRI, Käsmann L, Schönermarck U, Fischereder M, Grabmaier U, Manapov F, Rauch J, Orban M. Pembrolizumab-induced myocarditis in a patient with malignant mesothelioma: plasma exchange as a successful emerging therapy-case report. Transl Lung Cancer Res 2021;10(2):1039-1046. doi: 10.21037/tlcr-20-1095 


\section{Supplementary}

Table S1 Timeline of events from diagnosis of malignant mesothelioma to last follow-up

\begin{tabular}{|c|c|}
\hline Timeline & Patient diagnosed with malignant mesothelioma \\
\hline \multirow[t]{3}{*}{2 years prior to presentation } & $\begin{array}{l}\text { - Initial admission to hospital due to unknown weight loss (12 } \mathrm{kg} \text { in }<6 \text { months) and abdominal pain } \\
\text { - } \quad \text { PET-CT scan: supradiaphragmal, pleural tumor with diaphragmatic infiltration and suspicious } \\
\text { peritoneal lymph node involvement (cT4cN1 MX) }\end{array}$ \\
\hline & $\begin{array}{l}\text { - Cytoreductive surgery with HIPEC: partial diaphragmatic resection with alloplastic reconstruction, } \\
\text { subtotal parietal peritonectomy, with partial resection of the ribs 9-12, followed by HIPEC }\end{array}$ \\
\hline & - $\quad \mathrm{KPS} 90 \%$ \\
\hline 1 year prior to presentation & Recurrent disease with new pleural and perihepatic metastases, as assessed by PET-CT \\
\hline 9 months prior to presentation & $\begin{array}{l}\text { Next-generation sequencing was performed showing no targetable mutations using Oncomine Precision } \\
\text { Assay (Thermo Fisher Scientific, } 168 \text { Third Avenue Waltham, MA USA 02451). }\end{array}$ \\
\hline
\end{tabular}

3 months prior to presentation Progressive disease with multiple new bipulmonary and lymph node metastases with diffuse, extensive spread throughout the abdomen (PET-CT)

- Tumor board decides on the initiation of immunotherapy with anti-PD-L1 agent pembrolizumab

1 month prior to presentation Pembrolizumab (1st cycle)

1 week prior to presentation

Pembrolizumab (2nd cycle); onset of irAEs and hospital admission

Upon emergent presentation

Patient presents with generalized weakness, shortness of breath, double vision and ptosis

- $\quad$ Physical findings significant for $M G$

- $\quad$ Severely reduced EF ( $<35 \%)$; ST segment elevation in the posterior leads with polymorphic ventricular extrasystoles

- Elevated cardiac biomarkers

- $\quad$ Clinical criteria suggestive of $\mathrm{ICl}$-associated myocarditis

Day 1 to Day 10 following emergent presentation

Day 11 to Day 30 following emergent presentation

Day 30 following emergent presentation
Patient on i.v. methylprednisolone (100 mg/die)

Coronary artery disease ruled out by negative cardiac catheterization

Respiratory failure due to MG, patient is intubated

Upon clinical improvement, patient is extubated, NIV is continued due to hypercapnia

Patient develops arrhythmias with hemodynamic compromise (atrial fibrillation and flutter, ventricular tachyarrhythmias)

- Electrical and pharmacological cardioversion

- Reintubation and reventilation

- $\quad$ No remarkable improvement despite steroids

- Initiation of plasma exchange, clinical improvement and stabilization, steroid tampering, extubation

- $\quad$ EF recovery $(45-50 \%)$

Discharge to external weaning ward, oral prednisone, KPS 50\%

Table S1 (continued) 
Table S1 (continued)

\begin{tabular}{|c|c|}
\hline Timeline & Patient diagnosed with malignant mesothelioma \\
\hline $\begin{array}{l}6 \text { months after administration } \\
\text { of pembrolizumab }\end{array}$ & $\begin{array}{l}\text { Discharge from weaning ward and rehabilitation clinic to home; CT scan of thorax showing stable disease } \\
\text { according to RECIST, version } 1.1\end{array}$ \\
\hline \multicolumn{2}{|c|}{$\begin{array}{l}1 \text { year following administration Patient presents with dysphagia, KPS } 70 \% \\
\text { of pembrolizumab }\end{array}$} \\
\hline & $\begin{array}{l}\text { - Progressive disease (CT) with new pulmonary metastases and extrinsic compression of the } \\
\text { oesophagus }\end{array}$ \\
\hline & - $\quad$ EF appr. $50 \%$, sinus tachycardia with extrasystoles \\
\hline & - Initiation of palliative therapy (oesophagus stenting) \\
\hline & $\begin{array}{l}\text { Patient exhibits hypercapnia and respiratory failure, continues to deteriorate with Existus letalis due } \\
\text { to respiratory failure }\end{array}$ \\
\hline
\end{tabular}

PET-CT, positron emission tomography-computed tomography; HIPEC, heated intraperitoneal chemotherapy; KPS, Karnofsky performance status; PD-L1, programmed death-ligand 1; irAEs, immune-related adverse events; MG, myasthenia gravis; EF, left-ventricular ejection fraction as assessed by transthoracic echocardiography; ICl, immune-check-point inhibitors; NIV, non-invasive ventilation; RECIST, response evaluation criteria in solid tumors. 


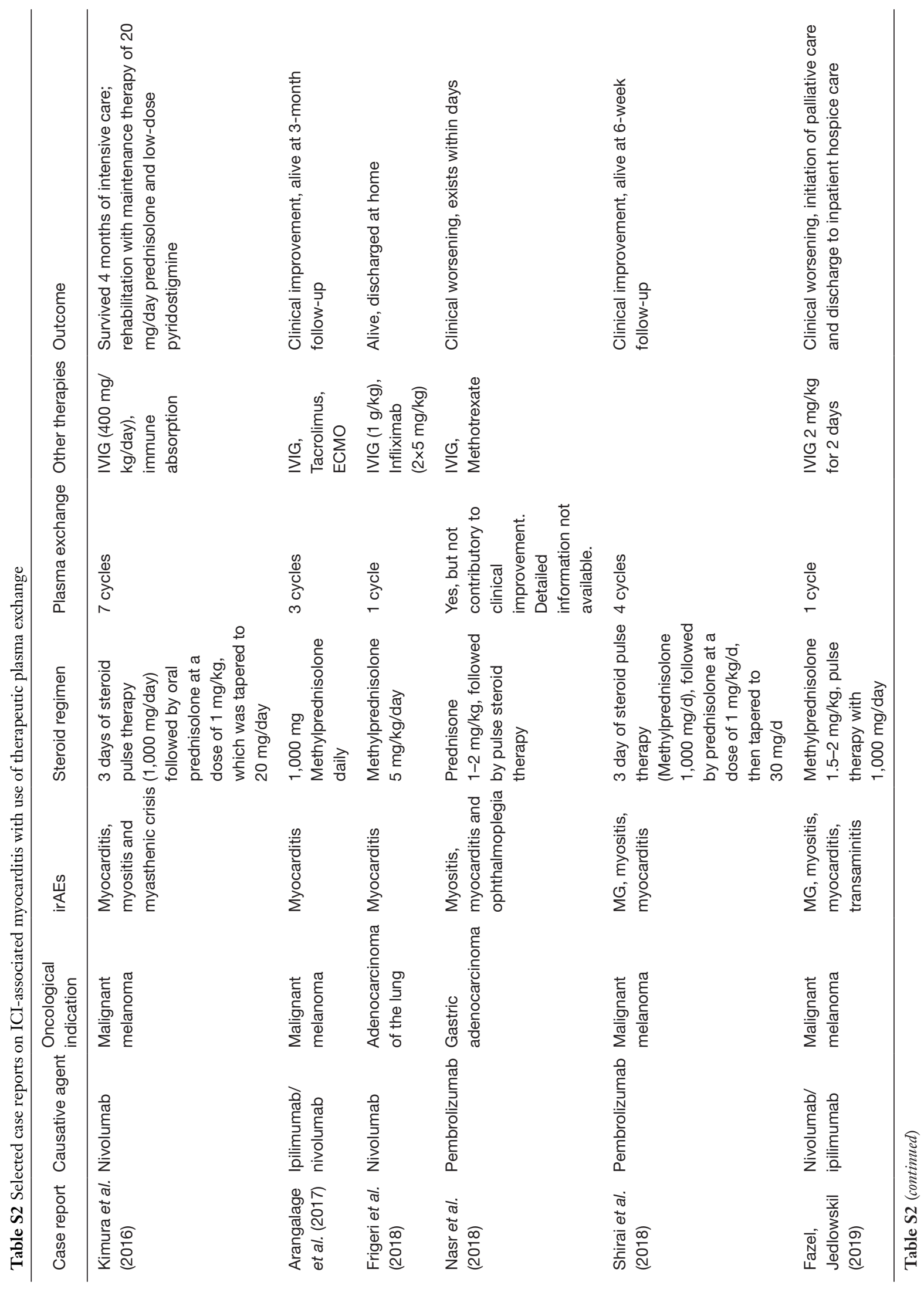




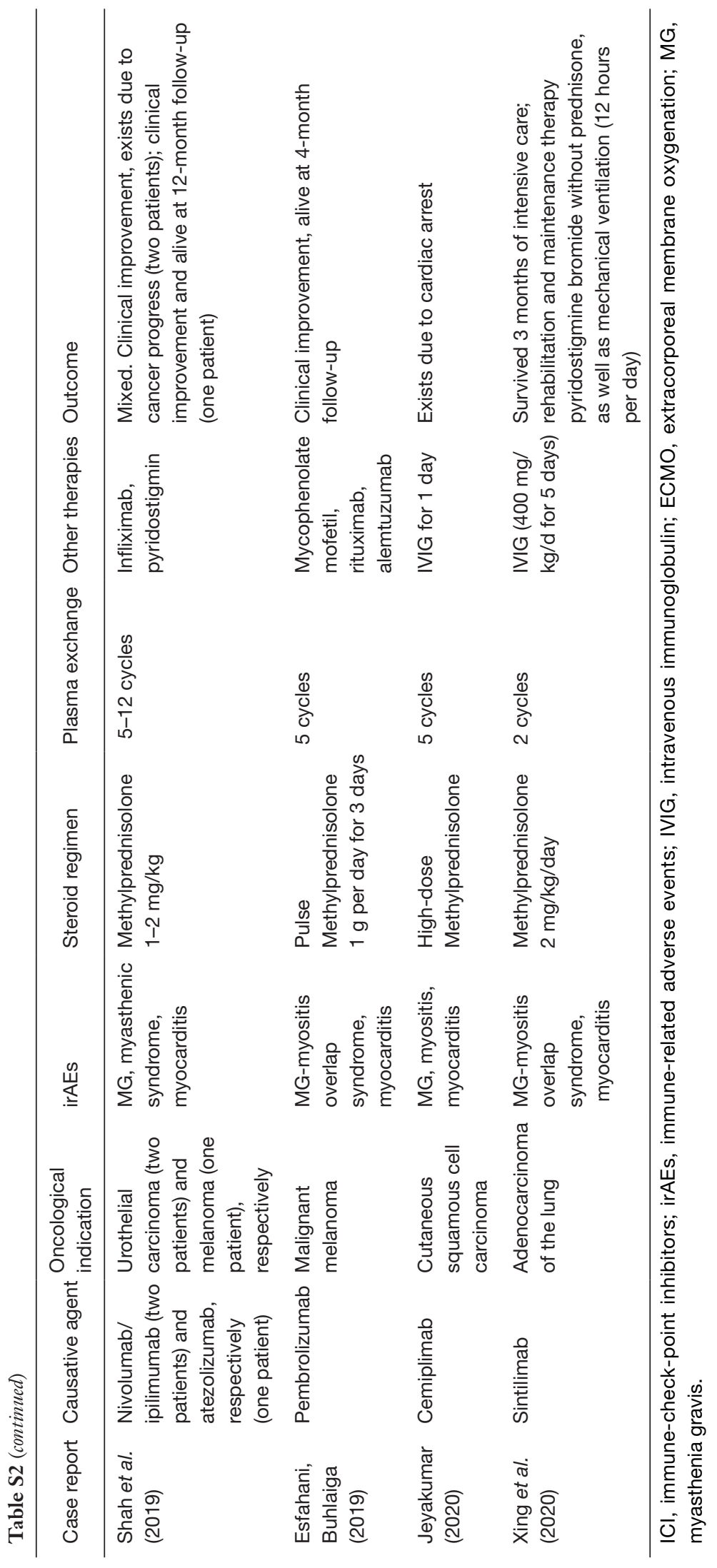

